\title{
Stakeholder involvement in the systematic optimisation of two school-based relationships and sex education interventions, Project Respect and Positive Choices
}

\author{
Ruth Ponsford, Jo Crichton, Rebecca Meiksin, Tara Tancred, Gemma Morgan, Nerissa Tilouche, Rona Campbell, Chris Bonell
}

Published Online November 22, 2018

University of Bristol, Bristol, UK (J Crichton PhD,

G Morgan PhD, P D); and London School of Hygiene \& Tropical Medicine, London, UK (R Ponsford PhD, R Meiksin MSc, TTancred PhD, NTilouche MA, Prof (Bonell PhD)

Correspondence to:

Dr Ruth Ponsford, London School of Hygiene \& Tropical Medicine, London WC1H 9SH,

ruth.ponsford@LSHTM.ac.uk

\section{Abstract}

Background There is increasing emphasis on involving intended beneficiaries and other stakeholders in the development of public health interventions to maximise acceptability and remove barriers to adoption, implementation, and maintenance before costly implementation. Yet the processes whereby key actors are engaged in intervention development are rarely reported, and frameworks for carrying out such work remain limited. We outline our approach to involving stakeholders in the optimisation of two school-based relationships and sex education programmes (Project Respect and Positive Choices) and reflect on the challenges of co-producing with teachers, students, and other partners.

Methods Systematic optimisation of both interventions involved a review of existing literature on effective approaches; consultation with staff and students on intervention content and delivery; drafting of intervention materials; further consultation with schools; and then intervention refinement in preparation for a pilot. Seven focus groups took place in southeast and southwest England involving 75 students aged 13-15 years and 22 school staff. A group of young people trained to advise on public health research were consulted on two occasions and a wide range of sexual health and sex education practitioners and policy makers shared their views at a stakeholder event.

Findings Consultation provided useful insights to inform intervention adaption in relation to who should deliver the programmes in schools; whether lessons should be taught in single sex classes; the format that guidance and lesson plans should take; the relevance and acceptability to students and teachers; and the need for the flexibility for materials to adapt to different school contexts. Genuine consultation and incorporation of school stakeholder views was challenging where stakeholder availability was limited and intervention development and implementation timelines were tight. Challenges also arose in relation to the weight to give divergent opinions among stakeholders and between stakeholders and researchers.

Interpretation Carrying out structured stakeholder engagement activities can yield valuable insights that can improve the applicability of interventions to local contexts before they are formally trialled. To genuinely engage stakeholders in intervention development requires sufficient time to both consult and adapt. In such consultations, it is important to attend not just to the voices of those who are the loudest and most powerful.

Funding National Institute for Health Research (NIHR).

Contributors

RP and JC contributed to collection, analysis, and interpretation of data, and led jointly on the writing of the abstract. RM contributed to collection, analysis, and interpretation of data, and the drafting of the abstract. TT, GM, and NT contributed to collection, analysis, and interpretation of data. RC contributed to the design and led on the Project Respect study for the southwest of England. CB conceived and led on both studies and contributed to the drafting of the abstract. All authors approved the final version of the abstract.

Declaration of interests

We declare no competing interests.

Acknowledgments

The views expressed are those of the authors and not necessarily those of the NHS, the NIHR or the Department of Health and Social Care. 